\title{
An asymptotic approach for shock-wave/transpired turbulent boundary layer interactions
}

By Atila P. Silva-Freire, University Engineering Dept. and Darwin College, Cambridge - England

\section{Introduction}

Recent technological advances, such as the development of supercritical wings, have considerably renewed interest in aircraft flight at transonic speeds. This type of wing is designed so that the change from the supersonic to the subsonic flow regions, which takes place on its surface as Mach number achieves its critical value, does not take place through a strong shock wave, as it does with wings of the conventional type, but with a weak shock wave. This greatly improves the aerofoil's performance since the shock wave/turbulent boundary layer interaction effects which often lead to flow separation and its detrimental consequences are largely minimized. Unfortunately, this type of aerofoil is very sensitive to small changes in Mach number and angle of attack and at off-design conditions, a strong enough shock wave may be produced on the surface resulting normally in a serious deterioration of the optimum performance.

With the objective of controling the shock wave/boundary layer interaction phenomenon and thereby preventing a deterioration of aerofoil performance, several techniques have been devised. These techniques include active means, such as tangential blowing and boundary layer suction, as well as passive means such as the introduction of a porous plate above a plenum chamber beneath the interaction.

The passive control technique has been studied in recent papers $[1,2,3,4,5]$ with promising results. It is based on the fact that the large pressure gradient over the interaction region provokes a natural flow circulation through the cavity from the region downstream to the region ahead of the shock wave. Thus the process combines injection upstream of the shock and suction downstream. This secondary flow thickens the boundary layer ahead of the shock wave which generates compression waves that coalesce and weaken the normal shock wave. These effects minimize the overall entropy rise across the shock and hence decrease the wave drag.

Since the majority of the studies on this technique have been conducted experimentally, and the few theoretical works $[4,5]$ have used some form of the 
integral-momentum equation to evaluate the skin-friction, which may be very inaccurate due to the difficulty in determining precisely the term $\mathrm{d} \theta / \mathrm{d} x$, it is of interest to develop a theory to explain the main features of this control technique by an alternative approach.

The aim of the present work is to formulate such a theory using perturbation techniques. The advantage of this approach is that analytical solutions can be obtained for the inner regions of the boundary layer. These solutions lead to a skin-friction equation which is much less sensitive than the integral-momentum equation to small changes in the flow parameters. In this work we are concerned with flows at high transonic speeds and extend the approach of Messiter [6] and Adamson \& Liou [7] for the permeable surface case.

The great practical difficulty of this method, however, is the quality of the surface. It is very difficult to obtain high porosity surfaces which at the same time have an acceptable level of roughness. In fact this difficulty is an important factor in determining the usefulness of this control technique since, as it is well known, roughness enhances separation. In order to overcome this problem several researchers have replaced the porous plate by perforated plates. This practice poses great complications to the problem of specifying the boundary conditions at the wall since a very complex flow develops in the vicinity of the openings. For this reason we assume in this work that the openings through which the fluid is blown or sucked are small relative to the boundary layer thickness and sufficiently close together that the mathematical boundary condition of homogeneous normal velocity at the wall can be used.

\section{The flow regions}

In the limiting case to be studied here Mach number is such that the normal shock wave penetrates deep into the boundary layer terminating close to the wall (Fig. 1). Hence the subsonic region is very thin and the upstream influence of the interaction region is negligible. The structure of the flow is illustrated in Fig. 1. This figure is similar to that given by Messiter [6] and Liou \& Adamson [7] since the disturbances caused to the main flow by the blown or sucked fluid are such that the various flow regions are preserved. This feature is best understood if one realizes that multi-layer theories have been developed to account for the fact that viscous terms, both turbulent and laminar, are important only in well defined regions of the flow and that this situation is not altered in any way by the blown or sucked fluid. Thus a classical two-deck structure is used in the upstream undisturbed region and a three-deck structure is used in the disturbed region downstream of the shock wave. Note that, although the flow configuration for this problem remains the same as for the solid plate problem, the expressions for the velocity profile will be quite different since the influence of the injected or sucked flow on the main flow has to be accounted for. Also several 


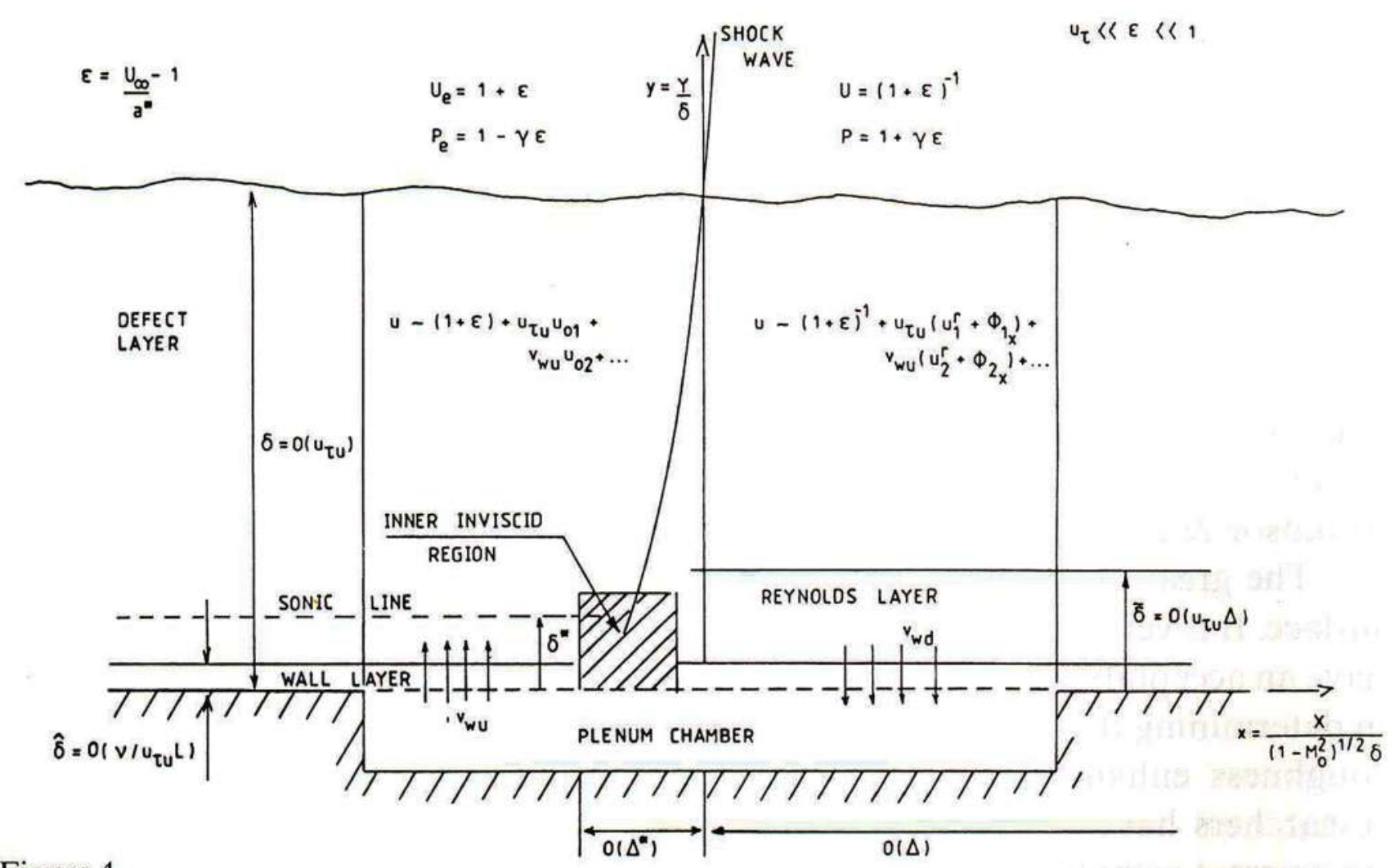

Figure 1

The flow regions.

important flow parameters such as the boundary layer thickness, $\delta$, and the friction velocity, $u_{\tau}$, will depend on the normal velocity at the wall.

The various flow regions quoted in Fig. 1 have been derived on the following physical grounds. Far away from the surface, in the undisturbed region, the moving fluid can be well described by inviscid equations. Approaching the wall a region, termed the defect region, is reached where turbulence becomes important. The equations of motion for this region must include, inertia, pressure and Reynolds stress terms. As the solution of the equations for this region cannot satisfy the no-slip condition at the wall a new layer needs to be considered, the wall layer. In this layer the inertia term due to the transpired fluid and the laminar stress term are the predominant terms in the momentum equation. The inclusion of this new layer permits the laminar viscosity to enforce a no-slip condition on the velocity profile. Let us now consider the disturbed region. In this region the inclusion of an inviscid and a wall layer is justified by the same arguments used for the undisturbed region. However, a marked difference from the two-deck model used upstream occurs here since now for most of the boundary layer the Reynolds stress is negligible when compared to the pressure gradient provoked by the shock wave. Thus there is a region in the disturbed region, the defect layer, which comprises most of the boundary layer and is governed by inviscid equation. Physically this means that no mechanism is available to transfer momentum from the wall layer to the defect layer and vice-versa. In order to solve this impasse a new region needs to be inserted between the defect and wall layers, the Reynolds layer. 
The order of magnitude of the various flow regions are shown in Fig. 1 with some of the relevant parameters of the problem. Note that, in addition to the small parameters normally used to represent the flow solution, i.e.,

$$
\varepsilon=\frac{U_{\infty}}{a^{*}}-1, \quad a^{*}=\begin{gathered}
\text { critical sounds speed in the external flow just ahead } \\
\text { of the shock }
\end{gathered}
$$

and

$$
u_{\tau}=\frac{1}{a^{*}} \sqrt{\frac{\tau_{w}}{\varrho_{w}},} \begin{aligned}
& \tau_{w}=\text { laminar stress at the wall } \\
& \varrho_{w}=\text { density at the wall }
\end{aligned}
$$

two new small parameters are introduced here, $v_{w u}$ and $v_{w d}$. The subscripts $w, u, d$ refer to conditions at the wall, upstream of the shock and downstream of the shock respectively.

In the next sections asymptotic expansions in terms of these four small parameters are used to represent the solutions of the various regions. Solutions for adjacent layers are then shown to match providing in this way a uniform solution for the whole flow field. Thus the length scales in Fig. 1 are determined from the substitution of the assumed asymptotic expansions into the equations of motion and from a careful analysis of the order of magnitude of the various resulting terms. Finally, since $\varepsilon \gg u_{\tau}$, the sonic line is located close to the wall and it is easily demonstrated [12] that $\Delta \gg \Delta^{*}$ and $\delta \gg \delta^{*}$, (see Fig. 1). Hence the inner inviscid flow region at the shock foot is exponentially small compared to the interaction region scaled by $\Delta$ and $\delta$.

\section{The initial profile}

The nature of the flow in the undisturbed region upstream of the shock has been extensively studied in references [8] and [9] where a theoretical analysis of the problem of transpired turbulent boundary layers has been performed. The analysis reveals that the flow has indeed a two-deck structure and proposes a new expression for the defect layer solution in place of the expressions of Simpson [10] and Stevenson [11]. Thus, according to those studies, the velocity defect solution made non-dimensional with respect to $a^{*}$ can be written as

$$
u_{u}=1+\varepsilon+u_{\tau u} u_{o 1}+v_{w u} u_{o 2}
$$

where

$$
u_{o 1}=\frac{1}{K} \ln y-\frac{\pi}{K}(2-w(y))
$$

and

$$
u_{o 2}=\frac{1}{4 K^{2}} \ln ^{2} y+\left(\frac{1}{2 K^{2}} \ln \frac{\delta}{\delta}+\frac{A_{u}}{2 K}\right) \ln y-\frac{\tilde{\pi}_{u}}{K}(2-w(y)) .
$$


The term $v_{w u} u_{o 2}$ in (1) accounts for the effects of the transpired fluid on the main flow. Note that as $v_{w u}$ tends to zero Eq. (1) reduces to the unblown expression used in references [6] and [7]. For this reason parameters $K, \pi$ and function $w(y)$ assume here their classical numerical values, that is

$$
\begin{aligned}
K & =0.41 \\
\pi & =0.55
\end{aligned}
$$

and

$$
w(y)=\text { Coles' wake function } .
$$

Here $y$ is the defect layer stretched co-ordinate $(=Y / \delta)$ and $\delta, \hat{\delta}$ denote the thickness of the defect and wall layers respectively. Parameters $A$ and $\tilde{\pi}$ in Eq. (3) are shown [9] to depend on the injection velocity and are given by

$$
A=5-512 v_{w 0}
$$

and

$$
\tilde{\pi}=-1.95 \ln \left|v_{w 0}\right|-3.1
$$

where

$v_{w 0}=$ characteristic normal velocity in the ovelap region.

The characteristic velocity has been determined in [9], assuming that the matching between the inner and outer regions is performed at $y=0.1$. Hence

$$
v_{w 0}=\frac{v(0.1)}{a^{*}} .
$$

This hypothesis was tested against a variety of flow conditions and is shown to give good results.

\section{The pressure distribution}

The analysis which follows is a natural extension of the work of Messiter [6] to the porous surface problem. Here, the equations governing the fluid motion in the defect layer do not include any turbulence terms since, as pointed out in $[6,12]$, these terms are so small when compared with the inertia and pressure terms that to the order considered here they can be neglected altogether. Furthermore, if the laminar stress and the entropy changes across the shock wave are neglected, it follows that the equations of motion for the defect layer can 
be written as

$$
\begin{aligned}
& a^{2} \operatorname{div} \boldsymbol{u}=\boldsymbol{u} \cdot \nabla \frac{u^{2}}{2} \\
& a^{2}=\frac{1}{2}(\gamma+1)-\frac{1}{2}(\gamma-1) \boldsymbol{u}^{2} \\
& \varrho \boldsymbol{u} \cdot \nabla \boldsymbol{u}=-\gamma^{-1} \nabla p
\end{aligned}
$$

and

$$
\boldsymbol{\Omega} \times \boldsymbol{u}=\gamma^{-1} t \nabla s
$$

In the above equations, the rectangular co-ordinates $X$ and $Y$ are made non-dimensional with respect to a geometric reference length such as, say, the distance from the shock to a leading edge. The velocity, $u$, and $a\left(=(p / \varrho)^{1 / 2}\right)$ are non-dimensionalized with respect to $a^{*}$. The non-dimensional pressure, density and temperature are all referred to their critical values just outside the boundary layer and ahead of the shock. In writing (13) we have assumed uniform total enthalpy.

From the gas dynamic equation and the vorticity equation, it can be shown that the length scale in the $x$-direction of the interaction region is $\Delta=0\left(b_{0} \delta\right)$, where $b_{0}^{2}=1-M_{0}^{2}\left(M_{0}=\right.$ Mach number in the external flow behind the shock wave). Hence the stretched co-ordinates for the defect layer are defined by

$$
x=\frac{X}{b_{0} \delta} \quad \text { and } \quad y=\frac{Y}{\delta}
$$

where

$$
b_{0}=(\gamma+1)^{1 / 2} \varepsilon^{1 / 2}\left[1-\frac{1}{4}(2 \gamma+1) \varepsilon+\cdots\right] .
$$

The solution is required to satisfy Eqs. (10) through (13) and appropriate boundary conditions determined by the normal shock wave jump conditions and the wall. As in Messiter's procedure we separate the asymptotic expansions for the solution into a rotational and an irrotational part. Hence it follows that the velocity profiles can be written as

$$
\begin{aligned}
u_{d}= & (1+\varepsilon)^{-1}+u_{\tau_{u}} u_{1}^{r}(x, y ; \varepsilon)+u_{\tau_{u}} \phi_{1_{x}}(x, y ; \varepsilon)+v_{w u} u_{2}^{r}(x, y ; \varepsilon) \\
& +v_{w u} \phi_{2_{x}}(x, y ; \varepsilon)
\end{aligned}
$$

and

$$
v_{d}=b_{0}\left[u_{\tau_{u}} \phi_{1_{x}}(x, y ; \varepsilon)+v_{w u} \phi_{2 y}(x, y ; \varepsilon)\right]+v_{w o d} v_{1}(x)
$$

where $v_{\text {wod }}$ is obtained from the inner layer solution.

In fact, as we shall see later, $v_{1}(x)$ is nearly constant and therefore without much loss of generality we take $v_{1}(x)=1$. The fact that the entropy is constant 
along a streamline together with the equation of state implies that along a steamline $p t^{-\gamma /(\gamma-1)} \sim p_{e} t_{u}^{-\gamma /(\gamma-1)}$. Thus, substitution of $t=a^{2}$ gives [6]

$$
p / p_{e}=1-\gamma\left(u-u_{u}\right)-\gamma^{2}\left(u_{u}-1\right)\left(u-u_{u}\right) .
$$

Also, from Crocco's theorem $\Omega \sim \gamma^{-1} p \mathrm{~d} s / \mathrm{d} \psi$ and hence $\Omega / p \sim \Omega_{u} / p_{e}$ along a streamline.

The rotational and irrotational terms of the different orders can now be obtained if expansions (16) and (17) are substituted into the vorticity and the gas dynamic equations, and the terms of same order of magnitude are collected. Note that, the fact that $u_{\tau}$ and $v_{w}$ are considered to be independent parameters greatly simplifies the process of grouping terms of same order.

On substituting expansions (16) and (17) into the vorticity equation, it follows that the rotational part of $u_{d}$ is given by

$$
u_{i}^{r}=\left(1+2 \gamma \varepsilon+\gamma(2 \gamma-1) \varepsilon^{2}+\cdots\right) u_{o i}(y), \quad i=1,2 .
$$

For the irrotational part of $u_{d}$ the governing equations are found to be

$$
\phi_{i_{x x}}+\phi_{i_{y y}}=0, \quad i=1,2 \text {. }
$$

It is easily shown that in a first approximation the shock wave is located at $x=0$, as indicated in Fig. 1. Thus Eqs. $(20 \mathrm{a}-\mathrm{b})$ are to be studied in the quarter plane $x \geqq 0, y \geqq 0$. The boundary conditions at $x=0$ are determined by the shock polar equations (Messiter, [6]) which reads

$$
v_{d}=\left(u_{u}-u_{d}\right)^{2} \frac{u_{u} u_{d}-1}{2 u_{u}^{2} /(\gamma+1)-\left(u_{u} u_{d}-1\right)} .
$$

Expanding $\phi_{1_{x}}$ in Eq. (21) in a Taylor series around $x=0$ gives

$$
\phi_{i_{x}}(0, y)=-2\left(1+(\gamma+1) \varepsilon+\left(\gamma\left(\gamma-\frac{1}{2}\right)+3\right) \varepsilon^{2}\right) u_{o i} .
$$

The boundary conditions for $y=0$ are given by $\phi_{i_{y}}(x, 0)=0, i=1,2$, and the requirement that all disturbances die out in the infinity. The general solution of Eqs. $(20 a-b)$ was first given in reference [12] and can be written as

$$
\begin{aligned}
\phi_{i}(x, y)= & -\frac{2}{\pi}\left\{1+(\gamma-1) \varepsilon+\left[\gamma\left(\gamma-\frac{1}{2}\right)+3\right] \varepsilon^{2}\right\} \\
& \cdot \int_{-\infty}^{\infty} u_{o i}(\eta) \ln \left\{x^{2}+(y-\eta)^{2}\right\}^{1 / 2} \mathrm{~d} \eta .
\end{aligned}
$$

In order to satisfy the boundary conditions at the wall $u_{o i}$ must be symmetric around $y=0$ and so we define $u_{o i}(-\eta)=u_{o i}(\eta)$. The potential part of the velocity profile is then given by

$$
\phi_{i_{x}}(x, y)=-\frac{4}{\pi}\left\{1+(\gamma-1) \varepsilon+\left[\gamma\left(\gamma-\frac{1}{2}\right)+3\right] \varepsilon^{2}\right\} \int_{0}^{\infty} \frac{x u_{o i}(y)}{x^{2}+(y-\eta)^{2}} \mathrm{~d} \eta .
$$


Substitution of expression (19) and (23) into Eq. (18) gives the pressure distribution across the boundary layer. We recall that although there are other regions between the defect layer and the wall, these other regions have vanishingly small $Y$-characteristic dimensions compared to the $Y$-characteristic dimension of the defect layer; hence to the order desired $\partial p / \partial y=0$ in these regions. Therefore, the limiting form of the defect layer pressure expression as $y \rightarrow 0$ gives the pressure at the wall, that is,

where

$$
\begin{aligned}
\frac{p_{w}}{p_{e}}(x)=1 & +\gamma\left\{2 \varepsilon+(2 \gamma-1) \varepsilon^{2}\right\} \\
& -\gamma\left\{1+\gamma \varepsilon+\varepsilon^{2}\left[(\gamma-1)^{2}+\frac{7 \gamma-7}{2}\right]\right\}\left\{u_{\tau_{u}} \phi_{1_{x}}(x, 0)+v_{w u} \phi_{2_{x}}(x, 0)\right\}
\end{aligned}
$$

$$
p_{e}=1-\gamma \varepsilon+\frac{\gamma}{2} \varepsilon^{2} .
$$

\section{The skin-friction equation}

This section is concerned with the study of the two inner layers, the Reynolds layer and the wall layer. From the fact that the solution of these two layers must match with the limiting form of the defect layer solution as $y \rightarrow 0$, it follows that the asymptotic expansion for the tangential velocity must depend on $u_{\tau u}$ and $v_{w u}$. Moreover, in these layers the shear stress terms which account for the momentum transfer between the wall and the main flow are significant and so we must expect the effects of suction to be strongly felt near the wall. As a result, the asymptotic expansions for these layers must also depend on $v_{w d}$.

As can be seen in Fig. 1 the length scales in the $Y$-direction are small compared to their characteristic lengths in $X$-direction. Consequently, standard turbulent boundary layer equations can be used here to describe the moving fluid in the inner regions.

Reynolds layer

The above remarks imply that for this region the governing equations can be written as

$$
\begin{aligned}
& \frac{\partial \varrho u}{\partial x}+\frac{\partial \varrho v}{\partial y}=0 \\
& \varrho u \frac{\partial u}{\partial x}+\varrho v \frac{\partial u}{\partial y}=\frac{-1}{\gamma} \frac{\partial p}{\partial x}+\frac{\partial}{\partial y}\left(\tau_{x y}+\frac{1}{R} \frac{\partial u}{\partial y}\right) \\
& \frac{\partial p}{\partial y}=0 \\
& t+\frac{\gamma-1}{2} u^{2}=\frac{\gamma+1}{2} \\
& p=\varrho t .
\end{aligned}
$$


These equations are the same as those presented in [7]. The $Y$-characteristic dimension of this region, $\tilde{\delta}$, is derived assuming that in the momentum equation the inertia terms are balanced by both the pressure and the Reynolds stress terms. Thus assuming that the Reynolds stress is $O\left(u_{\tau u}^{2}\right)$ it follows that $\tilde{\delta}=O\left(u_{\tau u} \Delta\right), \Delta=b_{0} \delta$.

The limiting form to which the Reynolds layer solution must match as $\tilde{y} \rightarrow \infty$ is obtained passing the limit as $y \rightarrow 0$ in the solution for the defect layer and then re-writing the resulting equation in terms of the Reynolds layer variable, $\tilde{y}$. This procedure gives

$$
\begin{aligned}
u(x, 0)= & (1+\varepsilon)^{-1}+u_{\tau u}\left[(1+2 \gamma \varepsilon+\cdots)\left(\frac{1}{K} \ln \frac{\tilde{\delta}}{\delta}+\tilde{u}_{o 1}\right)\right. \\
& \left.+(1+(\gamma-1) \varepsilon+\cdots) u_{1}(x)\right] \\
& +v_{w u}\left\{( 1 + 2 \gamma \varepsilon + \cdots ) \left[\frac{1}{4 K^{2}}\left(\ln \frac{\tilde{\delta}}{\delta}+\ln \tilde{y}\right)^{2}\right.\right. \\
& \left.+\left(\frac{1}{2 K^{2}} \ln ^{2} \frac{\delta}{\delta}+\frac{A_{u}}{2 K}\right)\left(\ln \frac{\tilde{\delta}}{\delta}+\ln \tilde{y}\right)-\frac{2}{K} \tilde{\pi}_{u}\right] \\
& \left.+(1+(\gamma-1) \varepsilon+\cdots) u_{2}(x)\right\}
\end{aligned}
$$

where

$$
\tilde{u}_{o 1}=\frac{1}{K} \ln \tilde{y}-\frac{2}{K} \pi
$$

and

$$
u_{i}(x)=-\frac{4 x}{\pi} \int_{0}^{\infty} \frac{u_{o i}(\eta)}{x^{2}+\eta^{2}} \mathrm{~d} \eta, \quad i=1,2 .
$$

The expressions above and the fact that the effects of suction must be accounted for in this region suggest that the expansion for $\tilde{u}$ is given by

$$
\begin{aligned}
\tilde{u}(x, \tilde{y})= & (1+\varepsilon)^{-1}+u_{\tau u}\left[\frac{1}{K} \ln \frac{\tilde{\delta}}{\delta}+\tilde{u}_{o 1}+\tilde{u}_{1}\right]+\varepsilon u_{\tau u}\left[\frac{1}{k} \ln \frac{\tilde{\delta}}{\delta} \tilde{u}_{1_{l}}+\tilde{u}_{11}\right] \\
& +v_{w u}\left[\left(\frac{1}{4 K^{2}} \ln ^{2} \frac{\delta}{\delta}+\frac{1}{2 K^{2}} \ln \frac{\delta}{\delta} \ln \frac{\tilde{\delta}}{\delta}+\frac{A_{u}}{2 K} \ln \frac{\tilde{\delta}}{\delta}-\frac{2 \tilde{\pi}_{u}}{K}\right)\right. \\
& \left.+\left(\frac{1}{2 K^{2}} \ln \frac{\tilde{\delta}}{\delta}+\frac{1}{2 K^{2}} \ln \frac{\delta}{\delta}+\frac{A_{u}}{2 K}\right) \ln \tilde{y}+\frac{1}{4 K^{2}} \ln ^{2} \tilde{y}+\tilde{u}_{2}\right] \\
& +v_{w u} \varepsilon\left[\ln 2 \frac{\tilde{\delta}}{\delta} \tilde{u}_{2 l}+\ln \frac{\tilde{\delta}}{\delta} \tilde{u}_{2 m}+\tilde{u}_{22}\right]+v_{w d}\left[\frac{1}{4 K^{2}} \ln ^{2} \tilde{y}\right. \\
& \left.+\left(\frac{1}{2 K^{2}} \ln \frac{\tilde{\delta}}{\tilde{\delta}}+\frac{A_{d}}{2 K}\right) \ln \tilde{y}-\frac{\tilde{\pi}_{d}}{K}(2-w(\tilde{y}))\right] .
\end{aligned}
$$


This equation clearly displays the effects of the upstream injection and of the downstream suction on the Reynolds layer solution. The asymptotic expression for the pressure in the Reynolds layer $\tilde{p}$ is given by

$$
\tilde{p}=\tilde{p}_{0}+u_{\tau u} \tilde{p}_{1}+\varepsilon u_{\tau u} \tilde{p}_{11}+v_{w u} \tilde{p}_{2}+\varepsilon v_{w u} \tilde{p}_{22}+\cdots .
$$

No terms of order $v_{w d}$ have been included in Eq. (31) since it has been demonstrated in [8] that the contributions of the suction to the pressure equation are of higher order. The corresponding expansions for the temperature and the density can be obtained through the energy and state equations.

To find a solution for the system of Eq. $(26 \mathrm{a}-\mathrm{e})$ we need first to choose a model for the Reynolds stress term in $(26 \mathrm{~b})$. Here we use the mixing length theory together with the hypothesis that the mixing length is proportional to the distance of the wall. Thus the Reynolds stress term reads

$$
\tau_{x y}=K^{2} \tilde{y}^{2}\left(\frac{\partial \tilde{u}}{\partial \tilde{y}}\right)^{2} .
$$

The equations of different order can now be determined by substituting the asymptotic expansions for the flow parameters and Eq. (32) into the equations of motion and collecting terms of same order. Then, from the momentum equation we get

$$
\begin{aligned}
& \frac{\partial \tilde{u}_{1}}{\partial x}=-\frac{1}{\gamma} \frac{\partial \tilde{p}_{1}}{\partial x}+\frac{\partial}{\partial \tilde{y}}\left(1+K \tilde{y} \frac{\partial \tilde{u}_{1}}{\partial \tilde{y}}\right)^{2} \\
& \frac{\partial \tilde{u}_{1_{l}}}{\partial x}=\frac{\partial}{\partial \tilde{y}}\left[2\left(K \tilde{y}+K^{2} \tilde{y}^{2} \frac{\partial \tilde{u}_{1}}{\partial \tilde{y}}\right) \frac{\partial \tilde{u}_{1}}{\partial \tilde{y}}\right] \\
& \frac{\partial \tilde{u}_{11}}{\partial x}=-\frac{1}{\gamma} \frac{\partial \tilde{p}_{11}}{\partial x}+\frac{\partial}{\partial \tilde{y}}\left[2\left(K \tilde{y}+K^{2} \tilde{y}^{2} \frac{\partial \tilde{u}_{1}}{\partial \tilde{y}}\right) \frac{\partial \tilde{u}_{11}}{\partial \tilde{y}}\right] \\
& \frac{\partial \tilde{u}_{2}}{\partial x}+\frac{\partial \tilde{u}_{o 1}}{\partial \tilde{y}}=-\frac{1}{\gamma} \frac{\partial \tilde{p}_{2}}{\partial x}+\frac{\partial}{\partial \tilde{y}}\left[\frac{1}{K} \ln \frac{\tilde{\delta}}{\delta}+\frac{1}{K} \ln \frac{\delta}{\delta}+A_{u}+\frac{1}{K} \ln \tilde{y}+2 K \tilde{y} \frac{\partial \tilde{u}_{2}}{\partial \tilde{y}}\right] \\
& \frac{\partial \tilde{u}_{2 l}}{\partial x}=\frac{\partial}{\partial \tilde{y}}\left(2 K \tilde{y} \frac{\partial \tilde{u}_{2 l}}{\partial \tilde{y}}\right) \\
& \frac{\partial \tilde{u}_{22}}{\partial x}+\frac{\partial}{K} \frac{\partial \tilde{u}_{11}}{\partial \tilde{y}}=\frac{\partial}{\partial \tilde{y}}\left(2 K \tilde{y} \frac{\partial \tilde{u}_{2 m}}{\partial \tilde{y}}\right) \\
& +\frac{\partial}{\partial \tilde{y}}\left[\frac{1}{K} \ln \tilde{y}+2 K \tilde{y} \frac{\partial \tilde{u}_{2}}{\partial \tilde{y}}\right]
\end{aligned}
$$


where the pressure terms of different order obtained from the defect layer solution are given by

$$
\begin{aligned}
\tilde{p}_{1}(x) & =-\gamma u_{1}(x) \\
\tilde{p}_{11}(x) & =-\gamma(\gamma-1) u_{1}(x) \\
\tilde{p}_{2}(x) & =-\gamma u_{2}(x) \\
\tilde{p}_{22}(x) & =-\gamma(\gamma-1) u_{2}(x)
\end{aligned}
$$

The pressure lowest order term $\tilde{p}_{0}$ is given by $\tilde{p}_{0}=1+\gamma \varepsilon+\cdots$

Equations $(33 a-c)$ and $(34 a-b)$ should be identical to the equations obtained by Liou \& Adamson [7] for the solid surface problem. However, expressions (33c) and (34b) do not agree with Liou and Adamson's corresponding equations. This discrepancy arises from the fact that in reference [7] the pressure expression was evaluated with the multiplication between $p_{e}$ and the right hand side terms of Eq. (25) not being performed whereas here we have correctly taken this operation into account. The effects of the transpiration on the main flow are represented by Eq. $(33 \mathrm{~d})$ through $g$. Now, the matching conditions and the fact that $p=p(x)$ imply that the solution of Eq. $(33 \mathrm{a})$ and $(33 \mathrm{~d})$ is

$$
\begin{aligned}
& \tilde{u}_{1}=\tilde{u}_{1}(x)=-\frac{\tilde{p}_{1}}{\gamma}(x)=u_{1}(x) \\
& \tilde{u}_{2}=\tilde{u}_{2}(x)=-\frac{\tilde{p}_{2}}{\gamma}(x)=u_{2}(x) .
\end{aligned}
$$

This result has already been used in writing (33c) and $(33 \mathrm{~g})$. The general form of the solutions of the unblown Eqs. $(33 \mathrm{~b}-\mathrm{c})$ was previously introduced in reference [13]. With that result one can show that the solution for Eqs. $(33 b-c)$ and $(33 \mathrm{e}-\mathrm{g})$ can be written as

$$
\begin{aligned}
& \tilde{u}_{1 l}=2 \gamma+\int_{0}^{x} \frac{B_{1 l}(\xi)}{x-\xi} \exp \left\{\frac{-\tilde{y}}{2 K(x-\xi)}\right\} \mathrm{d} \xi \\
& \tilde{u}_{11}=-\frac{1}{\gamma} \tilde{p}_{11}+2 \gamma \tilde{u}_{o 1}+\int_{0}^{x} \frac{B_{11}(\xi)}{x-\xi} \exp \left\{\frac{-\tilde{y}}{2 K(x-\xi)}\right\} \mathrm{d} \xi \\
& \tilde{u}_{2 l}=\frac{\gamma}{2 K^{2}}+\int_{0}^{x} \frac{B_{2 l}(\xi)}{x-\xi} \exp \left\{\frac{-\tilde{y}}{2 K(x-\xi)}\right\} \mathrm{d} \xi \\
& \tilde{u}_{2 m}=2 \gamma\left(\frac{1}{2 K^{2}} \ln \frac{\delta}{\delta}+\frac{A_{u}}{2 K}\right)+\int_{0}^{x} \frac{B_{2 m}(\xi)}{x-\xi} \exp \left\{\frac{-\tilde{y}}{2 K(x-\xi)}\right\} \mathrm{d} \xi
\end{aligned}
$$




$$
\begin{aligned}
\tilde{u}_{22}= & -\frac{1}{\gamma} \tilde{p}_{22}+2 \gamma \tilde{u}_{o 2}+\int_{0}^{x} \frac{B_{22}(\xi)}{x-\xi} \exp \left\{\frac{-\tilde{y}}{2 K(x-\xi)}\right\} \mathrm{d} \xi \\
& -\int_{0}^{x} \frac{B_{11}(\xi)}{2 K(x-\xi)} \ln (x-\xi) \exp \left\{\frac{-\tilde{y}}{2 K(x-\xi)}\right\} \mathrm{d} \xi
\end{aligned}
$$

where

$$
\tilde{u}_{o 2}=\frac{1}{4 K^{2}} \ln ^{2} \tilde{y}+\left(\frac{1}{2 K^{2}} \ln \frac{\delta}{\delta}+\frac{A_{u}}{2 K}\right) \ln \tilde{y}-\frac{2}{K} \tilde{\pi}_{u}
$$

and the $B$ 's are functions to be determined from conditions imposed by the matching of the defect and wall layer solutions. In writing $(36 \mathrm{c})$ we have anticipated that $B_{1_{l}}=0$. One can easily see that the above equations do match with the defect layer solution, since as $\tilde{y}$ tends to infinity each integral term above tends to zero exponentially and the remaining terms substituted into Eq. (30) yield an expression which does match with Eq. (27). Also, as $x$ tends to zero the solution satisfies the shock wave jump conditions.

The defect layer solution as $\tilde{y}$ tends to zero must match with the inner layer solution as the inner layer variable $\hat{y}$ tends to infinity. So, it is opportune at this point to study the asymptotic behavior of the integral terms in Eqs. $(35 \mathrm{~b}-\mathrm{c})$ and $(36 \mathrm{~b}-\mathrm{d})$ as $\tilde{y}$ tends to zero. Thus, according to reference [13] it follows that

$$
\begin{aligned}
& \int_{0}^{x} \frac{B_{i}(\xi)}{x-\xi} \exp \left\{\frac{-\tilde{y}}{2 K(x-\xi)}\right\} \mathrm{d} \xi \sim-B_{i}(x) \ln \frac{\tilde{y}}{2 K}+g_{i}(x) \\
& g_{i}(x)=B_{i}(x)\left[\ln x-\gamma_{e}\right]
\end{aligned}
$$

where $\gamma_{e}=0.57721$. The second integral term on the right hand side of Eq. (36d) occurs only in the porous surface problem and one can show that

$$
\begin{aligned}
& \int_{0}^{x} \frac{B_{i}(\xi)}{x-\xi} \ln (x-\xi) \exp \left\{\frac{-\tilde{y}}{2 K(x-\xi)}\right\} \mathrm{d} \xi \sim B_{i}(x) \ln ^{2} \frac{\tilde{y}}{2 K}+g_{i}^{\prime}(x) \\
& g_{i}^{\prime}=B_{i}(x)\left[-\frac{1}{2} \ln ^{2} x-\gamma_{e}^{\prime}+\gamma_{e}\right]
\end{aligned}
$$

where

$$
\gamma_{e}^{\prime}=\frac{1}{2} \int_{0}^{\infty} \ln ^{2} u \exp (-u) \mathrm{d} u
$$


Finally, if expressions (35-36) are substituted into Eq. (30), and the limit as $\tilde{y}$ tends to zero is taken, it follows that

$$
\begin{aligned}
\tilde{u}(x, \tilde{y})= & (1+\varepsilon)^{-1}+u_{\tau u}\left\{\frac{1}{K} \ln \frac{\tilde{\delta}}{\delta}+\tilde{u}_{o 1}+\tilde{u}_{1}(x)\right\} \\
& +\varepsilon u_{\tau u}\left\{\frac{1}{K} \ln \frac{\tilde{\delta}}{\delta}\left[2 \gamma+B_{1_{l}}\left(-\ln \frac{\tilde{y}}{2 K}+\ln x-\gamma_{e}\right)\right]\right. \\
& \left.+\left[(\gamma-1) u_{1}+2 \gamma \tilde{u}_{o 1}+B_{11}\left(-\ln \frac{\tilde{y}}{2 K}+\ln x-\gamma_{e}\right)\right]\right\} \\
& +v_{w u}\left\{\frac{1}{4 K^{2}} \ln ^{2} \frac{\tilde{\delta}}{\delta}+\frac{1}{2 K^{2}} \ln \frac{\delta}{\delta} \ln \frac{\tilde{\delta}}{\delta}+\frac{A_{u}}{2 K} \ln \frac{\tilde{\delta}}{\delta}-\frac{2}{K} \tilde{\pi}_{u}\right. \\
& \left.\left.+\left(\frac{1}{2 K^{2}} \ln \frac{\tilde{\delta}}{\delta}+\frac{1}{2 K^{2}} \ln \frac{\delta}{\delta}+\frac{A_{u}}{2 K}\right) \ln \tilde{y}+\frac{1}{4 K^{2}} \ln \tilde{y}^{2}+u_{2}(x)\right]\right\} \\
& +v_{w u} \varepsilon\left\{\ln 2 \frac{\tilde{\delta}}{\delta}\left[\frac{\gamma}{2 K^{2}}+B_{2_{l}}\left(-\ln \frac{\tilde{y}}{2 K}+\ln x-\gamma_{e}\right)\right]\right. \\
& +\ln \frac{\tilde{\delta}}{\delta}\left[2 \gamma\left(\frac{1}{2 K^{2}} \ln \frac{\delta}{\delta}+\frac{A_{u}}{2 K}\right)+B_{2 m}\left(-\ln \frac{\tilde{y}}{2 K}+\ln x-\gamma_{e}\right)\right] \\
& +\left[(\gamma-1) u_{2}(x)+2 \gamma \tilde{u}_{o 2}+B_{22}\left(-\ln \frac{\tilde{y}}{2 K}+\ln x-\gamma_{e}\right)\right. \\
& \left.\left.+B_{11}\left(-\frac{1}{2} \ln \frac{\tilde{y}}{2 K}+\frac{1}{2} \ln { }^{2} x+\gamma_{e}^{\prime}-\gamma_{e}\right)\right]\right\} \\
& {\left[\frac{1}{4 K^{2}} \ln \ln ^{2} \tilde{y}+\left(\frac{1}{2 K^{2}} \ln \frac{\tilde{\delta}}{\delta}+\frac{A_{d}}{2 K}\right) \ln \tilde{y}-\frac{\tilde{\pi} \tilde{x}_{d}}{K}(2-w(\tilde{y}))\right] . }
\end{aligned}
$$

Wall layer

Since the no-slip condition is not satisfied by the defect solution, it becomes necessary to introduce a new layer adjacent to the wall to satisfy this condition, the so-called wall layer. Thus in this layer viscous effects must be such that the fluid is brought to rest at the wall. Moreover, it is well known, on both physical and theoretical grounds, that in most of the wall layer the turbulent and laminar stress terms balance each other in the $x$-momentum equation. Hence if we assume that the Reynolds stress is $O\left(u_{\tau u}^{2}\right)$, it follows that

$$
\frac{1}{R_{w}} \frac{\delta}{\delta} \frac{\partial \hat{u}}{\partial \hat{y}}=O\left(u_{\tau u}^{2}\right)
$$


where the circumflex indicates a wall layer quantity and $R_{w}$ is the Reynolds number based on properties evaluated at the wall condition. Then, anticipating that $\hat{u}=O\left(u_{\tau u}\right)$, we have

$$
\hat{\delta}=\delta / u_{\tau u} R_{w}
$$

and so the stretched co-ordinate for this region is

$$
\hat{y}=y u_{\tau u} R_{w} .
$$

Now from the fact that, as $\hat{y}$ tends to infinity the Reynolds stress term becomes much more significant than the laminar stress term and from the form of Eq. (41), we are led to assume that the tangential velocity profile in the overlap region between the defect and the wall layers can be written as

$$
\hat{u}(x, \hat{y})=u_{\tau d}(x) \hat{u}_{o 1}(\hat{y})+v_{w u} \hat{u}_{o 2}(\hat{y})+v_{w d} \hat{u}_{o 3}(\hat{y})
$$

where $u_{\tau d}$ is in general a function of $x, \varepsilon, u_{\tau u}, v_{w u}, v_{w d}, \delta, \tilde{\delta}$, and $\delta$; $\hat{u}_{o 1}$ represents a logarithm function with the form of Eq. (2) and $\hat{u}_{o 2}$ and $\hat{u}_{o 3}$ represent bilogarithm functions with the form of Eq. (3). In fact, the idea here is to separate $u_{\tau d}(x)$ in two parts: one to account for the shock and upstream injection effects and another to account for the downstream suction effects. Hence the velocity profile in this layer is written as

$$
\begin{aligned}
\hat{u}= & u_{\tau u}\left(1+a_{1} \varepsilon+a_{2} \varepsilon^{2}+u_{\tau u} \hat{u}_{1}(x)+\varepsilon u_{\tau u} \frac{1}{K} \ln \frac{\tilde{\delta}}{\delta} \hat{u}_{1_{l}}(x)+\varepsilon u_{\tau u} \hat{u}_{11}(x)\right. \\
& +v_{w u} \hat{u}_{2}(x)+v_{w u} \varepsilon \ln ^{2} \frac{\tilde{\delta}}{\delta} \hat{u}_{2_{l}}(x)+v_{w u} \varepsilon \ln \frac{\tilde{\delta}}{\delta} \hat{u}_{2 m}(x) \\
& \left.+v_{w u} \varepsilon \hat{u}_{22}(x)+\cdots\right)\left(\frac{1}{K} \ln \hat{y}+A_{u}\right)+v_{w u}\left(1+\varepsilon \hat{v}_{2}(x)+\varepsilon^{2} \hat{v}_{3}(x)+\cdots\right) \\
& +\left[\frac{1}{4 K^{2}} \ln ^{2} \hat{y}+\frac{A_{u}}{2 K} \ln \hat{y}+\frac{A_{u}^{2}}{4}+\hat{v}_{1}(x)\right]+u_{\tau s}\left(\frac{1}{K} \ln \hat{y}+A_{d}\right) \\
& +v_{w d}\left[\frac{1}{4 K^{2}} \ln ^{2} \hat{y}+\frac{A_{d}}{2 K} \ln \hat{y}+\frac{A_{d}^{2}}{4}\right] .
\end{aligned}
$$

Now, since the wall and the defect layer solution must match in an overlap domain, it follows that the value of the unknown functions and parameters can be determined if Eqs. (41) and (45) are compared term by term in this overlap region. 
Thus from the matching conditions we have

$$
\begin{aligned}
& 1=u_{\tau u}\left(\frac{1}{K} \ln \frac{\delta}{\delta}+A_{u}+\frac{2}{K} \pi\right)+v_{w u}\left(\frac{1}{4 K^{2}} \ln ^{2} \frac{\delta}{\hat{\delta}}+\frac{A_{u}}{2 K} \ln \frac{\delta}{\delta}+\frac{A_{u}^{2}}{4}+\frac{2 \tilde{\pi}_{u}}{K}\right) \\
& 0=u_{\tau s}\left(\frac{1}{K} \ln \frac{\tilde{\delta}}{\delta}+A_{d}\right)+v_{w d}\left(\frac{1}{4 K^{2}} \ln ^{2} \frac{\tilde{\delta}}{\delta}+\frac{A_{d}}{2 K} \ln \frac{\tilde{\delta}}{\bar{\delta}}+\frac{A_{d}^{2}}{4}+\frac{2 \tilde{\pi}_{d}}{K}\right) \\
& -1=a_{1} u_{\tau u}\left(\frac{1}{K} \ln \frac{\delta}{\hat{\delta}}+A_{u}\right)+\hat{v}_{2}(x) v_{w u}\left(\frac{1}{4 K^{2}} \ln ^{2} \frac{\delta}{\hat{\delta}}+\frac{A_{u}}{2 K} \ln \frac{\delta}{\delta}+\frac{A_{u}^{2}}{4}\right) \\
& \frac{1}{2}=a_{2} u_{\tau u}\left(\frac{1}{K} \ln \frac{\delta}{\hat{\delta}}+A_{u}\right)+\hat{v}_{3}(x) v_{w u}\left(\frac{1}{4 K^{2}} \ln ^{2} \frac{\delta}{\hat{\delta}}+\frac{A_{u}}{2 K} \ln \frac{\delta}{\hat{\delta}}+\frac{A_{u}^{2}}{4}\right) \\
& F \hat{u}_{1}(x)=u_{1}(x), \quad\left(\text { here } F=u_{\tau u}\left(\frac{1}{K} \ln \frac{\delta}{\hat{\delta}}+A_{u}\right)\right) \\
& F \hat{u}_{1_{l}}(x)+a_{1}=2 \gamma+B_{1_{l}}\left(-\ln \frac{\tilde{y}}{2 K}+\ln x+\gamma_{e}\right) \\
& F \hat{u}_{11}(x)+a_{1} \frac{1}{K} \ln \tilde{y}=(\gamma-1) u_{1}(x)+2 \gamma \tilde{u}_{o 1} \\
& +B_{11}\left(-\ln \frac{\tilde{y}}{2 K}+\ln x-\gamma_{e}\right) \\
& F \hat{u}_{2_{l}}(x)=\frac{\gamma}{2 K^{2}}+B_{2_{l}}\left(-\ln \frac{\tilde{y}}{2 K}+\ln x-\gamma_{e}\right) \\
& F \hat{u}_{2 m}(x)=2 \gamma\left(\frac{1}{2 K^{2}} \ln \frac{\delta}{\delta}+\frac{A_{u}}{2 K}\right)+B_{2 m}\left(-\ln \frac{\tilde{y}}{2 K}+\ln x-\gamma_{e}\right) \\
& \hat{v}_{2}(x) \tilde{u}_{o 2}+F \hat{u}_{22}=(\gamma-1) u_{2}+2 \gamma \tilde{u}_{o 2}+B_{22}\left(-\ln \frac{\tilde{y}}{2 K}+\ln x-\gamma_{e}\right) \\
& +B_{11}\left(-\frac{1}{2} \ln ^{2} \frac{\tilde{y}}{2 K}+\frac{1}{2} \ln ^{2} x+\gamma_{e}^{\prime}-\gamma_{e}\right) \text {. }
\end{aligned}
$$


The solutions of the above equations are

$$
\begin{aligned}
& a_{1}=-\frac{1}{F}\left\{1+v_{w u}\left[2 \gamma(1-2 K)+2 K a_{1}\right]\left[\frac{1}{4 K^{2}} \ln ^{2} \frac{\delta}{\delta}+\frac{A_{u}}{2 K} \ln \frac{\delta}{\delta}+\frac{A_{u}^{2}}{4}\right]\right\} \\
& a_{2}=1 / 2 F \\
& \hat{u}_{1}=u_{1} / F \\
& B_{1_{l}}=0 \\
& \hat{u}_{1_{l}}=\left(2 \gamma-a_{1}\right) / F \\
& B_{11}=\left(2 \gamma-a_{1}\right) / K \\
& \hat{u}_{11}=\frac{1}{F}\left[(\gamma-1) u_{1}-\frac{4 \gamma \pi}{K}+B_{11}\left(\ln 2 K x-\gamma_{e}\right)\right] \\
& \hat{u}_{2}=u_{2} / F \\
& B_{2_{l}}=0 \\
& \hat{u}_{2_{l}}=2 \gamma /\left(4 K^{2} F\right) \\
& B_{2 m}=0 \\
& \hat{v}_{1}=0 \\
& \hat{u}_{2 m}=\frac{2}{F} \gamma\left(\frac{1}{2 K^{2}} \ln \frac{\delta}{\delta}+\frac{A_{u}}{2 K}\right) \\
& \hat{v}_{2}=2 \gamma(1-2 K)+2 K a_{1} \\
& B_{22}=\frac{2 \gamma-a_{1}}{K} \ln 2 K+2 K\left(2 \gamma-a_{1}\right)\left(\frac{A_{u}}{2 K}+\frac{1}{2 K^{2}} \ln \frac{\delta}{\delta}\right) \\
& =
\end{aligned}
$$

An expression for the skin friction can now be determined if expressions (47a) through $\mathrm{p}$ are substituted into Eq. (45). Thus the resulting 
expression is

$$
\begin{aligned}
u_{\tau d}(x)= & u_{\tau u}\left\{1+a_{1} \varepsilon+a_{2} \varepsilon^{2}+u_{\tau u} \frac{u_{1}}{F}+\varepsilon u_{\tau u} \frac{1}{K} \ln \frac{\tilde{\delta}\left(2 \gamma-a_{1}\right)}{F}\right. \\
& +\varepsilon u_{\tau u} \frac{1}{F}\left[(\gamma-1) u_{1}-\frac{4 \gamma \pi}{K}+\frac{\left(2 \gamma-a_{1}\right)}{K}\left(\ln 2 K x-\gamma_{e}\right)\right]+v_{w u} \frac{u_{2}}{F} \\
& +v_{w u} \varepsilon \ln ^{2} \frac{\delta}{\delta} \frac{\gamma}{2 K^{2} F}+v_{w u} \varepsilon \ln \frac{\tilde{\delta}}{\delta} \frac{2}{F} \gamma\left(\frac{1}{2 K^{2}} \ln \frac{\delta}{\delta}+\frac{A_{u}}{2 K}\right) \\
& +v_{u w} \varepsilon \frac{1}{F}\left[2 K\left(2 \gamma-a_{1}\right) \frac{2 \tilde{\pi}_{u}}{K}+(\gamma-1) u_{2}+\left(\frac{2 \gamma-a_{1}}{K} \ln 2 k\right.\right. \\
& \left.+2 K\left(2 \gamma-a_{1}\right)\left(\frac{A_{u}}{2 K}+\frac{1}{2 K^{2}} \ln \frac{\delta}{\delta}\right)\right)\left(\ln 2 K+\ln x-\gamma_{e}\right) \\
& \left.\left.+\frac{2 \gamma-a_{1}}{K}\left(-\frac{1}{2} \ln ^{2} 2 K+\frac{1}{2} \ln ^{2} x+\gamma_{e}^{\prime}-\gamma_{e}\right)\right]\right\}+u_{\tau s}
\end{aligned}
$$

where $a_{1}$ and $a_{2}$ can be obtained through Eqs. $(47 \mathrm{a}-\mathrm{b})$.

In deriving the above equations we have used the same procedure as for the undisturbed case; that is, expression (48) is determined by conditions arising from the matching of the Reynolds and the wall layers. The above equation seems to be very complicated but this complication is unavoidable and arises from the fact that this equation has to account for all the disturbances on the flow.

\section{The boundary conditions at the wall}

In order to obtain a solution for the entire flow field we need to couple the solution for the external flow established in the previous sections with the solution for the flow inside the plenum chamber. However, this coupling is not always easily carried out since the boundary condition to be prescribed at the wall is very complex. This is particularly true if the fluid is blown or sucked through orifices or slots. Here to simplify the calculations we assume that the velocity of the flow though the plate's surface is directly proportional to the pressure difference between the external and the internal regions. So, the velocity at the wall is given by

$$
v_{w}(x)=\sigma\left(p_{w}-p_{h}\right)
$$

where

$$
\begin{aligned}
p_{w} & =\text { external pressure } \\
p_{h} & =\text { internal pressure } \\
\sigma & =\text { plate factor }
\end{aligned}
$$


Furthermore we assume that the pressure inside the cavity which results from the mutual interaction between the external flow and the flow inside the plenum chamber is constant. The net mass flow rate over the porous region is zero, therefore

$$
\int_{L} \varrho_{w} v_{w} \mathrm{~d} x=0
$$

where

$L=$ length of the porous bed.

For a surface of constant porosity, substitution of (49) into (50) gives

$$
p_{h}=\int_{L} \varrho_{w} p_{w}(x) \mathrm{d} x / \int_{L} \varrho_{w} \mathrm{~d} x
$$

where $p_{w}(x)$ is given by expression (25).

The normal velocity at the wall is then determined by Eqs. (49) and (51). Equations (51) may at first sight appear to be an oversimplification of the problem but any attempt to develop a more sophisticated solution would lead to great complications without any significant gain in accuracy.

\section{Numerical results}

Before considering the interaction with flow through a porous surface we will first compare our results with measured data on a solid surface.

Despite the importance of the problem of shock wave/boundary layer interaction, the quantity of data which is available in the literature for this phenomenon for flows at transonic speeds is very limited. The main reason for this is the great degree of complication involved in the planning and execution of such experiments. Indeed, some practical problems, such as the control of the position of the shock, are normally very difficult to solve and hence great care must be taken during the reduction of the experimental data. The analysis of the data for this type of problem is therefore a very delicate and laborious process. Repeatability is also very difficult to accomplish due to the difficulty in resetting the shock wave in the same position for distinct runs. Moreover, the shock usually has a random movement around a mean point and so most of the measured values presented in the literature are in fact mean values.

It should also be noted that the theory developed in this work corresponds to the case of shock wave/boundary layer interactions in two-dimensional, non-separated flow. Thus, it is necessary to be sure that any experimental data set to which this theory is compared satisfies these two conditions. A survey of the literature indicates that the results which seem to be the most adequate for testing the theory are those of Gadd [14] and Sawyer and Long [15]. Even so the results of Gadd were obtained in a tube of small diameter with a strong pressure 
gradient and so his flow conditions do not reproduce faithfully the flat plate conditions assumed to hold here. The results of Sawyer and Long for $M=1.27$ and 1.37 are certainly two-dimensional and non-separated; however their flow conditions are not exactly those desired here since a $\lambda$-foot structure develops in the interaction region. Furthermore, the conditions downstream of their shock never reach the asymptotic levels predicted by the shock wave jump conditions even at great distances downstream of the shock. However, to this author's knowledge this work is the most detailed experimental analysis of shock wave/boundary layer interaction for this Mach number range. For this reason, and despite all the discrepancies between the experimental and theoretical flow conditions, we have here decided to compare our theoretical predictions with this work.

The equations presented in sections IV through VI complemented by the momentum equation, which provides an additional relation for $u_{\tau}$ and $\delta$, are solved numerically. Initial values of $\delta(x), u_{\tau}(x)$ and $v_{w}(x)$ are fixed. With these values the expressions for the velocity and for the pressure can be substituted into the momentum equation to give new values of $\delta$. Next the porous bed equation is solved to give new values of $v_{w}$. The skin-friction equation is then solved to give new values of $u_{\tau}$. The process is repeated until these values converge to the solution of the problem. For the inviscid inner region, which was by-passed in the previous sections, a solution is obtained patching the solutions upstream and downstream of the shock wave. In order to do that an estimate of the length of this inner region is required. Here we assume that the non-dimensional length of this region is determined by the points $x_{u}=-5$ and $x_{d}=3.5$ as suggested by Gadd's data.

The flow conditions for the solid surface case are shown in Table 1. A comparison between the theoretical predictions and the experimental data is presented in Figs. $(2 a-b)$ and $(3 a-b)$. As can be seen, the agreement is quite reasonable. Note that, although the theoretical pressure profile is higher than the experimental profile, both these results have the same overall trend. The difficulty with the skin-friction results is that the theoretical solution downstream of the shock wave increases very slowly with $x$ and so it does not represent well enough the recovery of $C_{f}$ to its undisturbed values downstream of the shock. However the prediction of the minimum value of $C_{f}$ is very impressive which does suggest the usefulness of the proposed theory.

Table 1

Experimental flow conditions.

\begin{tabular}{lllll}
\hline conditions & $M$ & $\varepsilon$ & $R /$ foot & $\delta_{0}\left(x=-20^{\prime \prime}\right)$ \\
\hline$a$ & 1.270 & 0.214 & $1.12 \times 10^{6}$ & $1.55^{\prime \prime}$ \\
$b$ & 1.370 & 0.292 & $1.12 \times 10^{6}$ & $1.43^{\prime \prime}$ \\
\hline
\end{tabular}




\section{Pressure Distribution}

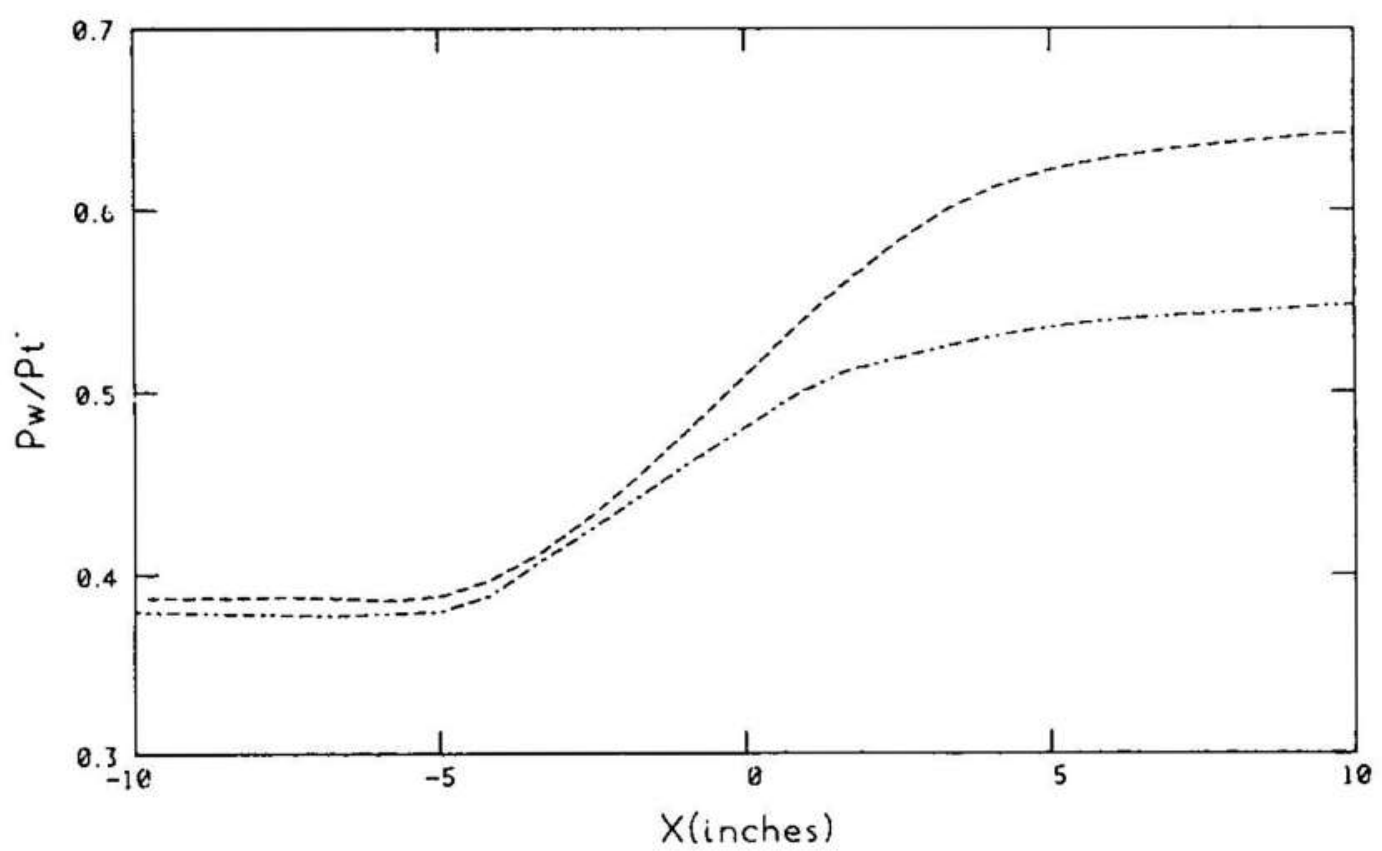

Figure 2a

Results for solid plate, $M=1.27 .--\cdots--$, experiment; ---, theory.

Friction Coefficient

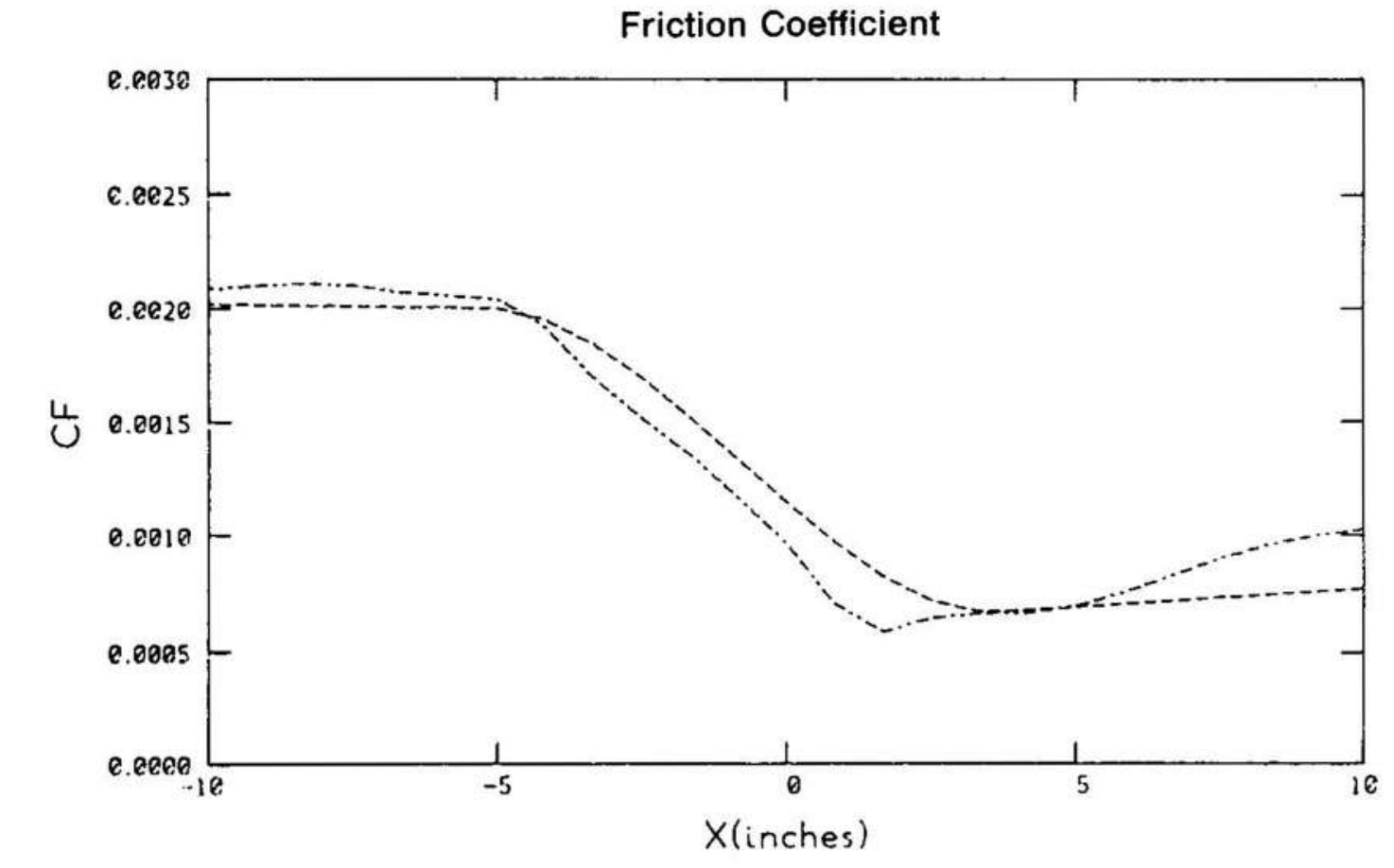

Figure $2 b$

Results for solid plate, $M=1.27 .--\cdots--$, experiment; --- , theory.

No experimental data was found for the porous surface case which entirely fulfilled the theoretical assumptions of this work. Hence, in order to study the effects of a porous surface on the properties of a shock wave/boundary layer interaction, we just replaced part of the surface of Sawyer and Long's experiments by a porous plate with a chamber beneath. The plenum chamber is 
Pressure Distribution

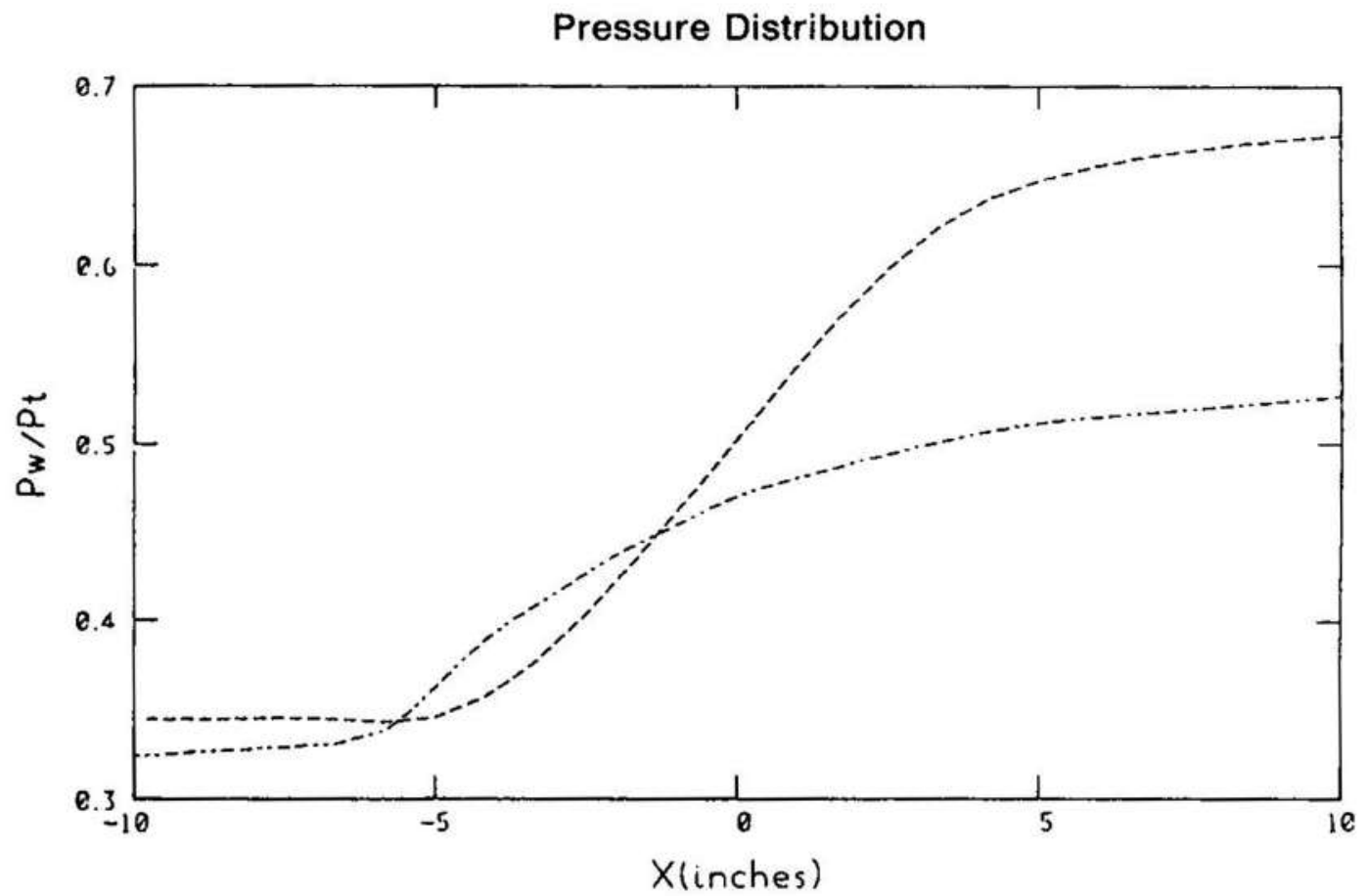

Figure $3 \mathrm{a}$

Results for solid plate, $M=1.37 .--\cdots--$, experiment; ---, theory.

Friction Coefficient

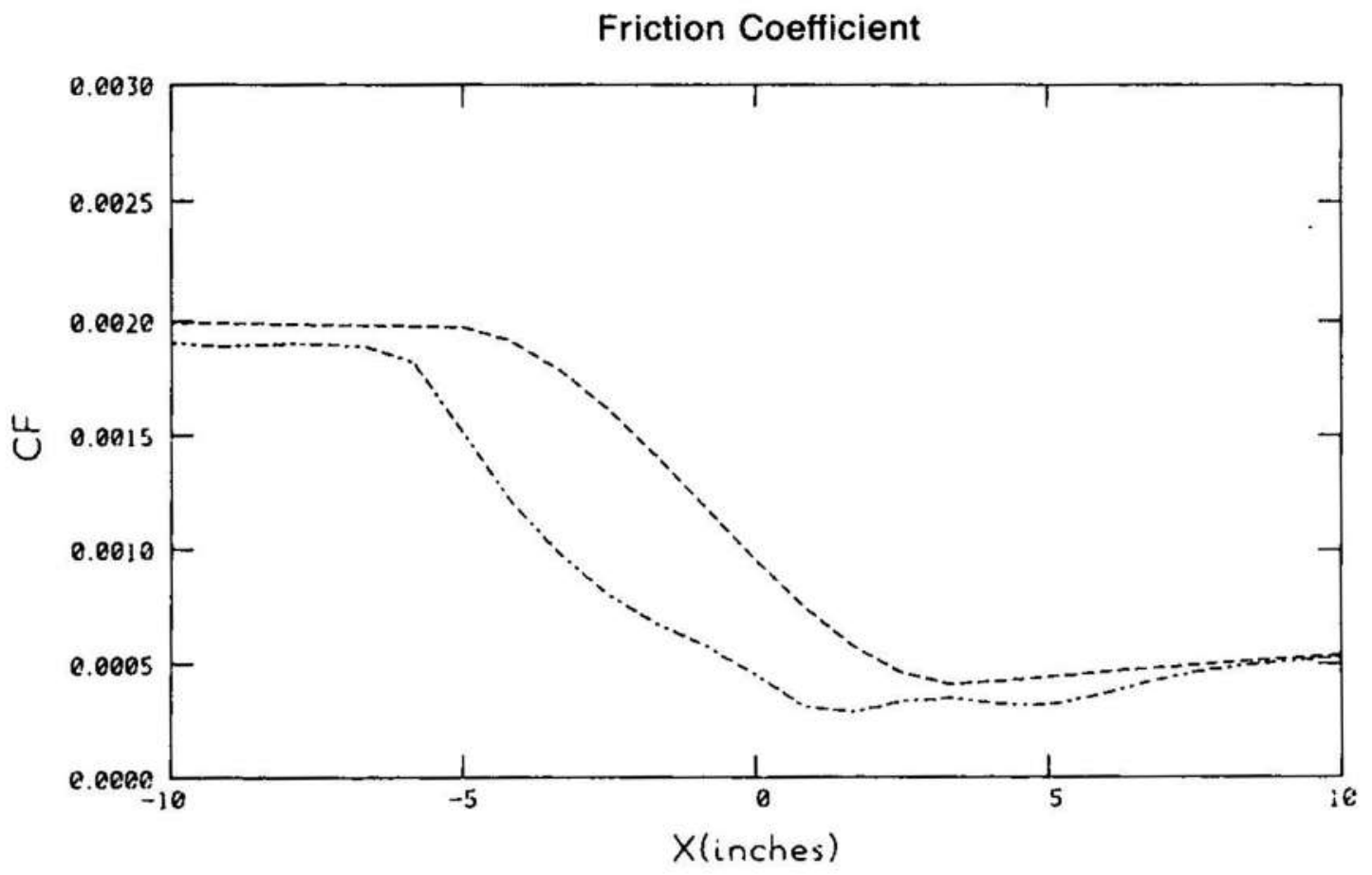

Figure $3 b$

Results for solid plate, $M=1.37 .--\cdots--$, experiment; ---, theory.

characterized by its porosity and dimensions. Combinations of these two parameters give a wide range of conditions to be studied. To illustrate the possible cases of interest, we consider here porosities which yield weak $\left(\sigma=0.005, v_{w}=0.001\right)$ and moderate injection rates $\left(\sigma=0.02, v_{w}=0.004\right)$ and long $\left(=40\right.$ inches $\left.\sim 24 \delta_{0}\right)$ and short $\left(=10\right.$ inches $\left.\sim 6 \delta_{0}\right)$ stretches of porous 


\section{Pressure Distribution}

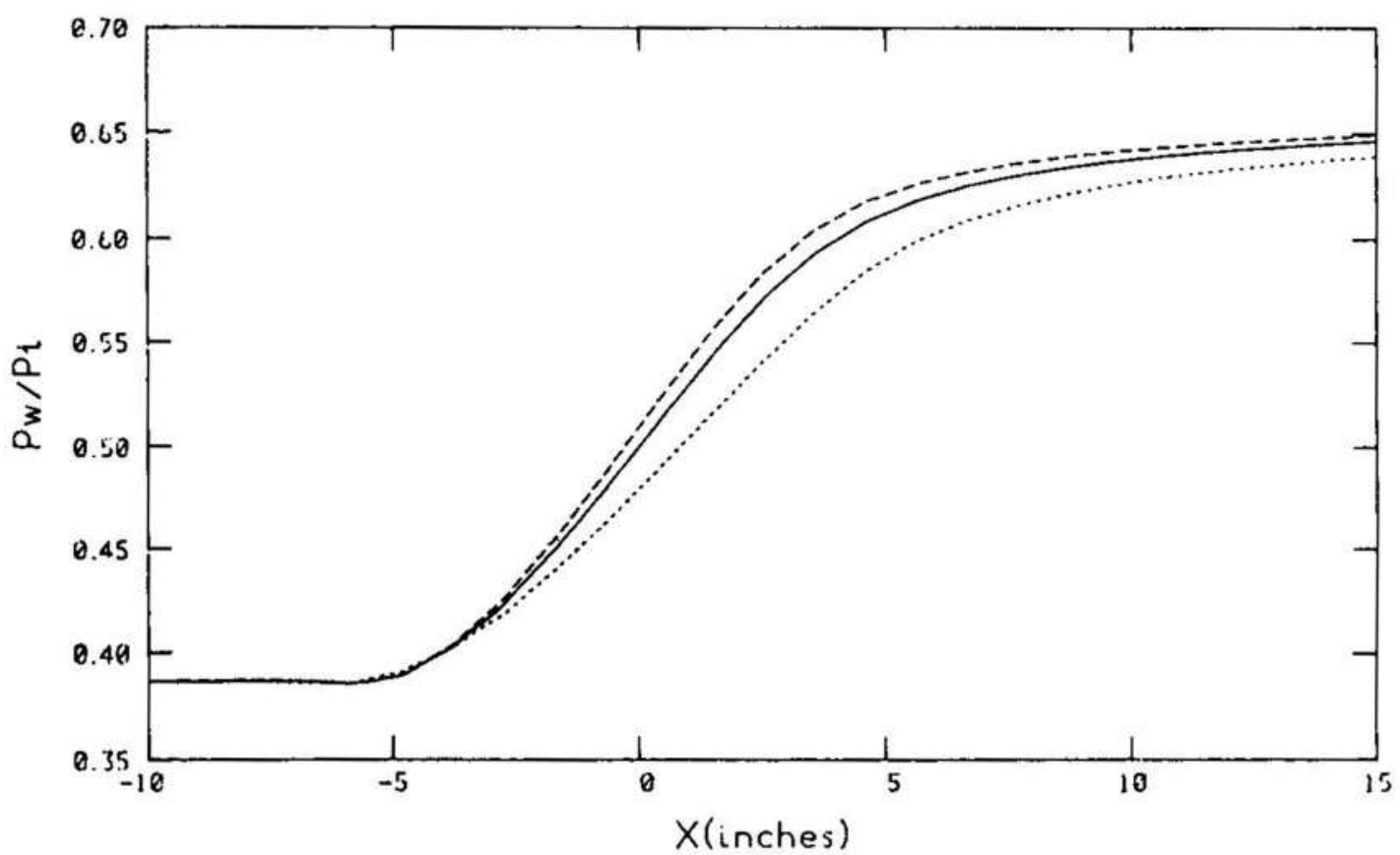

Figure $4 \mathrm{a}$

Results for a long stretch of porous surface, $M=1.27$. - - $\sigma=0.0 ;-, \sigma=0.005 ; \cdots, \sigma=0.02$.

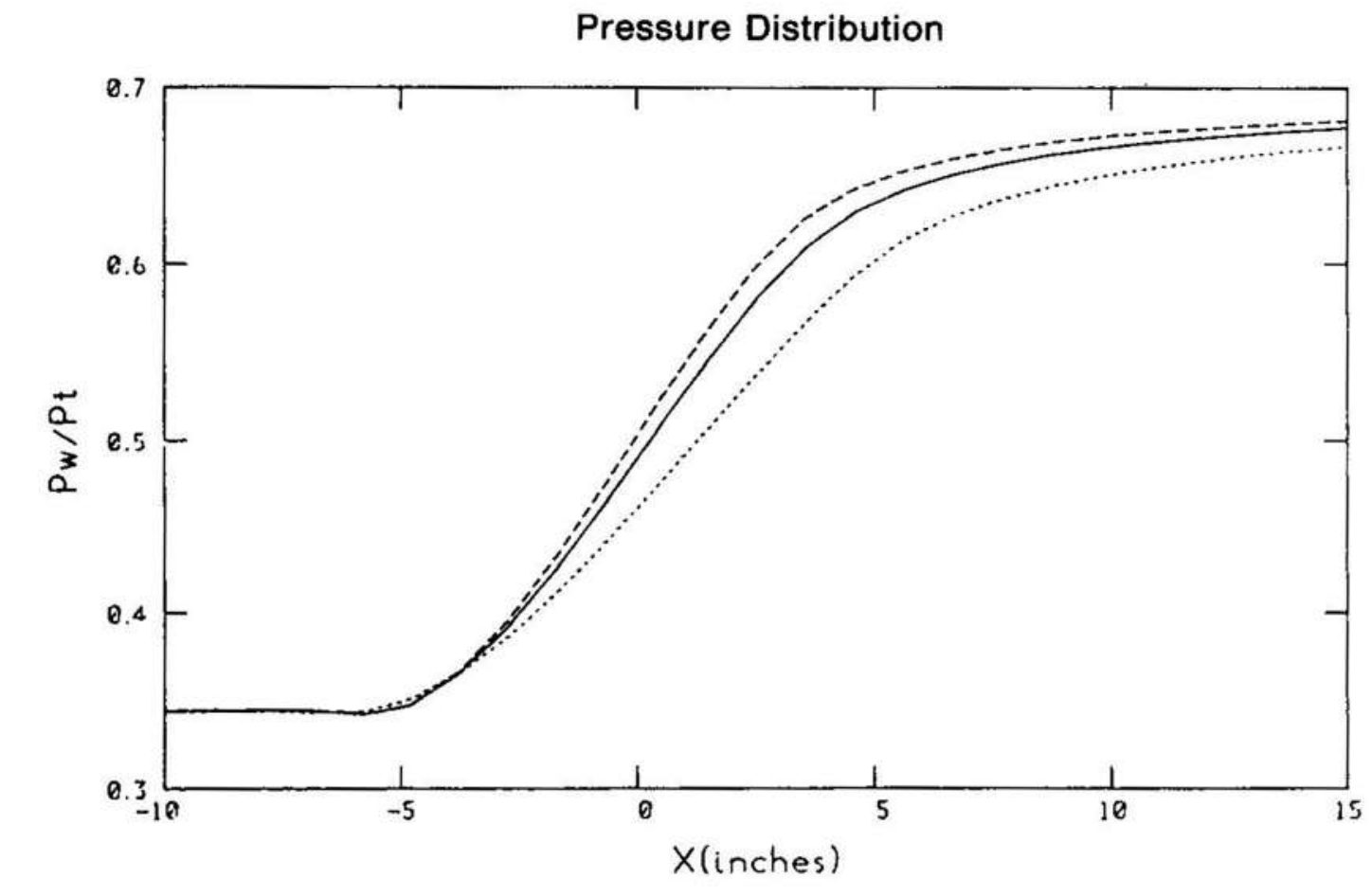

Figure $4 b$

Results for a long stretch of porous surface, $M=1.37$. ---, $\sigma=0.0 ;-, \sigma=0.005 ; \cdots, \sigma=0.02$.

surfaces. The typical plenum chamber effect on the wall pressure distribution is shown in Figs. $(4 \mathrm{a}-\mathrm{b})$ for a long stretch of porous surface. Note that, although the changes in pressure for moderate injection rates are significant, they are not dramatic. The changes in the skin-friction behaviour are shown in Figs. $(5 a-b)$ and $(6 a-b)$. As can be seen, moderate injection and suction 


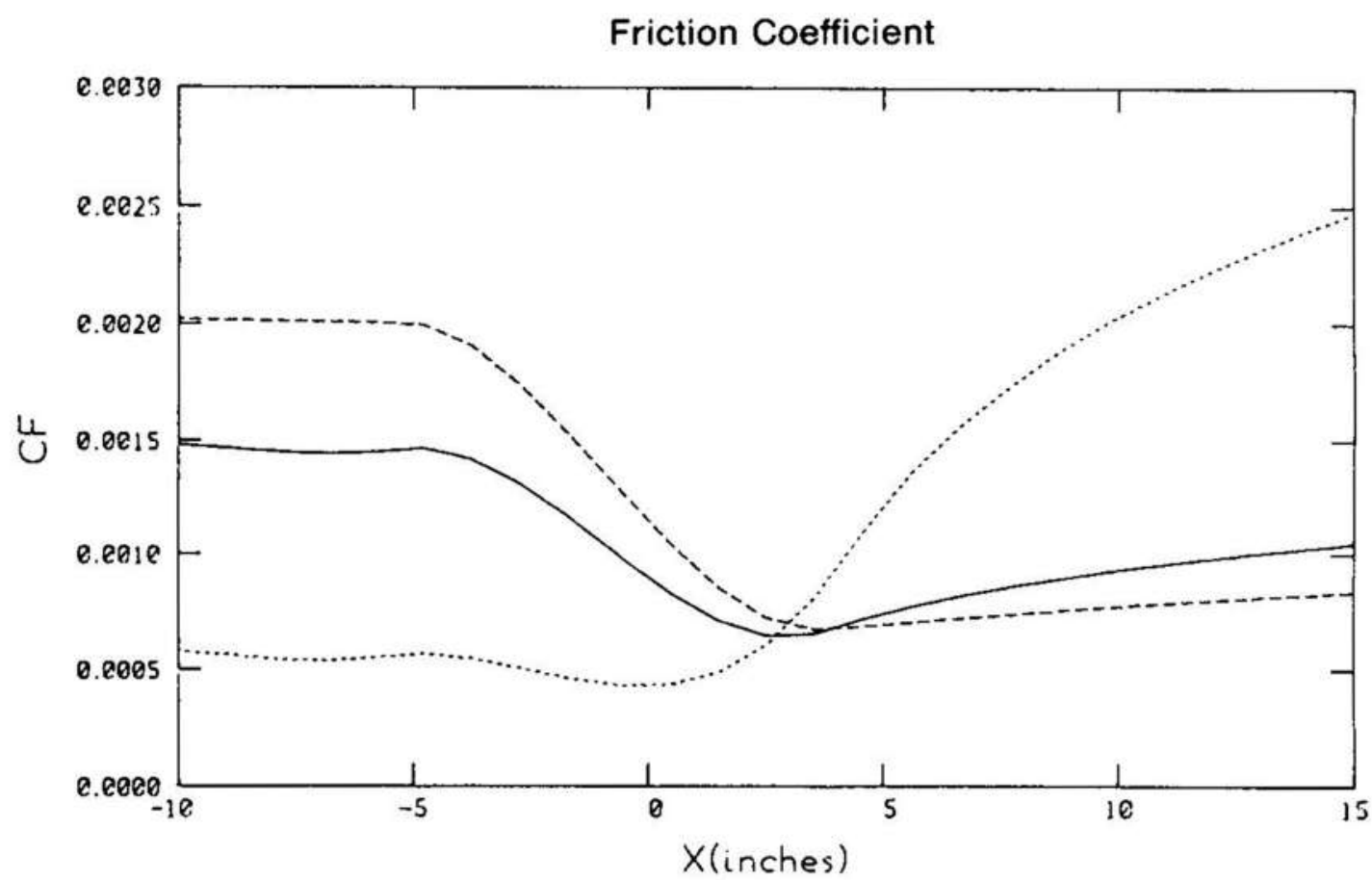

Figure $5 \mathrm{a}$

Results for a long stretch of porous surface, $M=1.27$. - - $\sigma=0.0 ;-, \sigma=0.005 ; \cdots, \sigma=0.02$.

\section{Friction Coefficient}

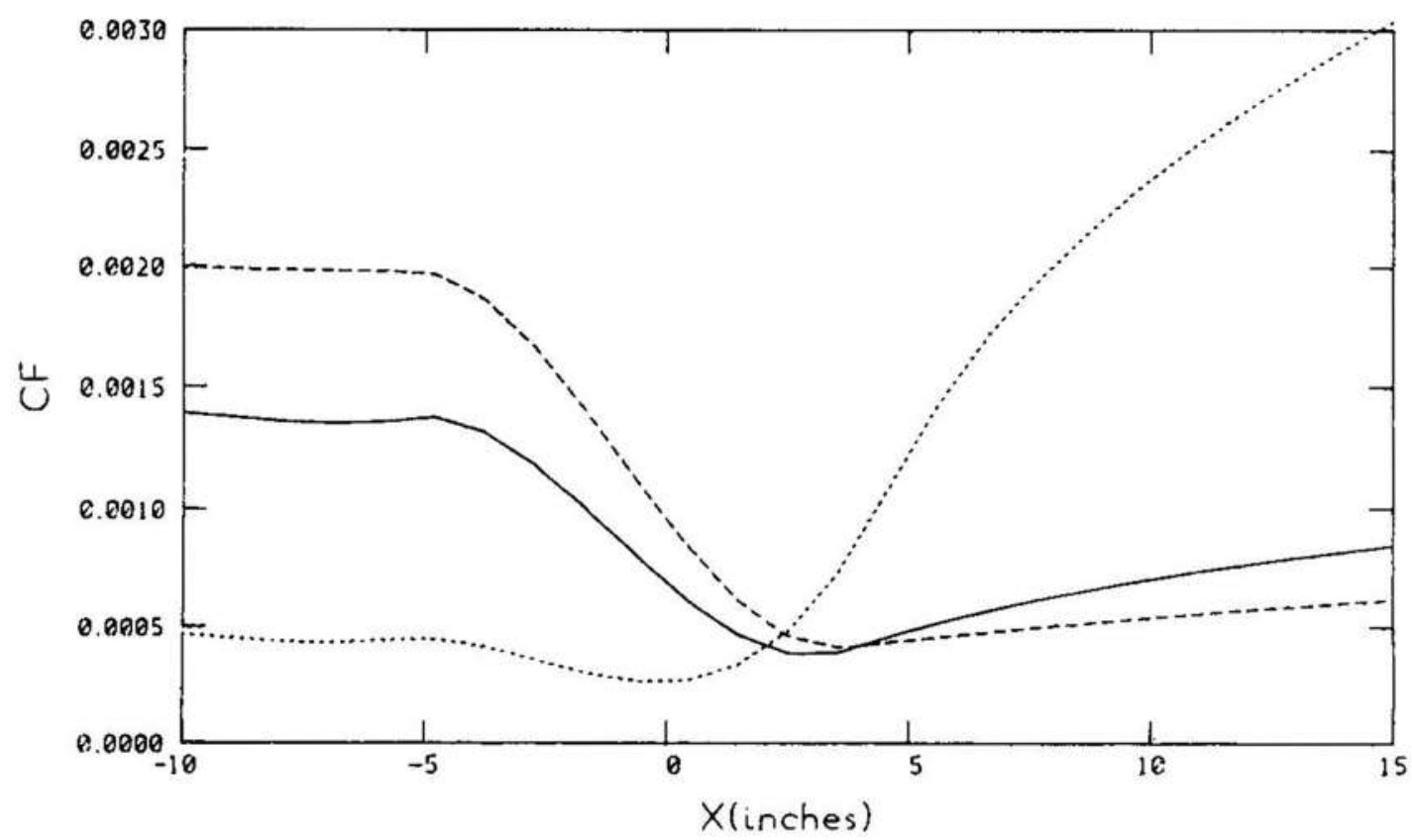

Figure 5b

Results for a long stretch of porous surface, $M=1.37$. ---, $\sigma=0.0 ;-, \sigma=0.005 ; \cdots, \sigma=0.02$.

rates result in a significant increase in $C_{f}$ downstream of the shock. Thus, these figures suggest that, if the problems of practical arrangement and optimization of a bleed system like the one studied here can be solved, separation will almost certainly be prevented. 


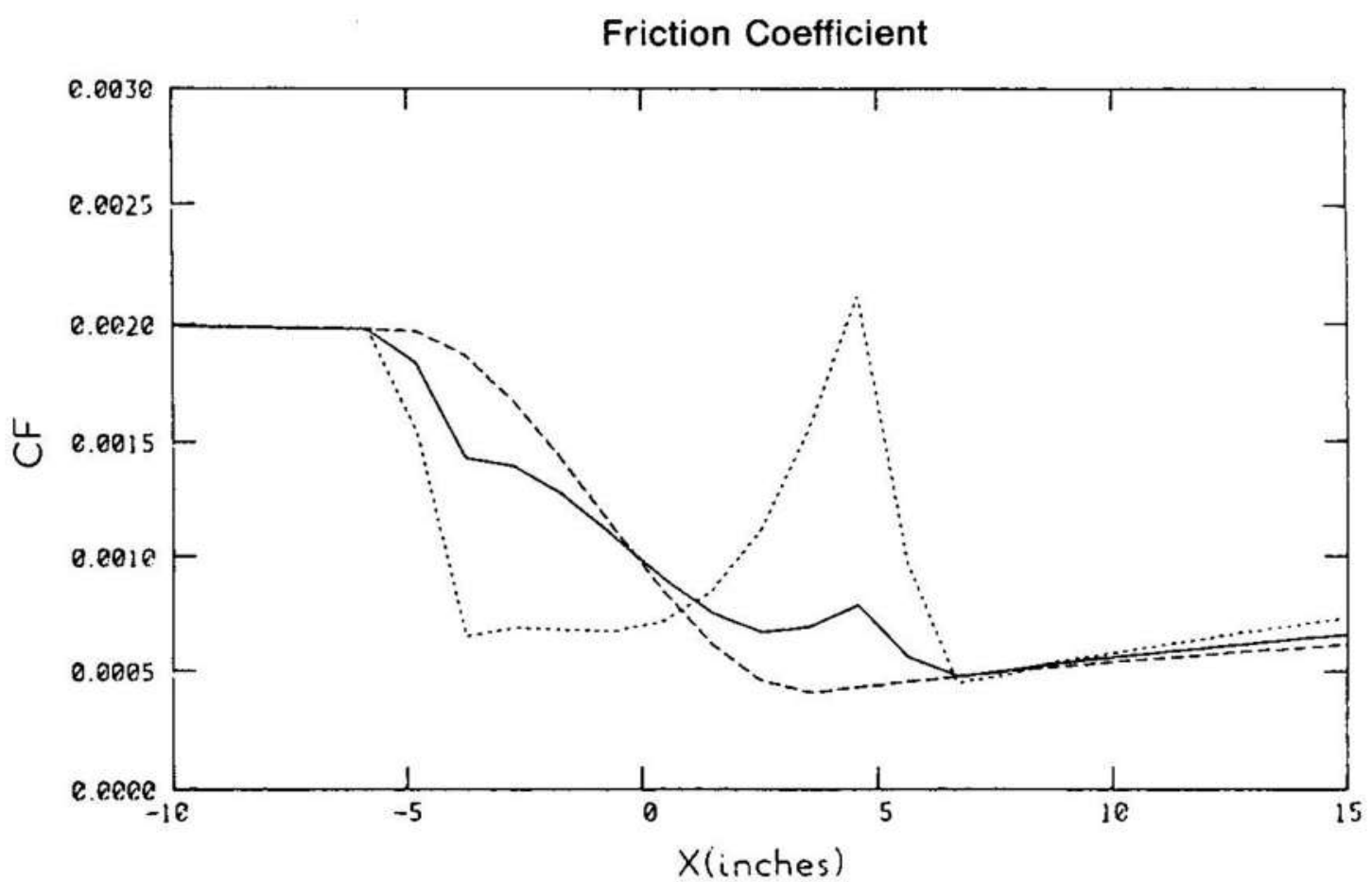

Figure 6a

Results for a short stretch of porous surface, $M=1.27 .---, \sigma=0.0 ;-, \sigma=0.005 ; \cdots, \sigma=0.02$.

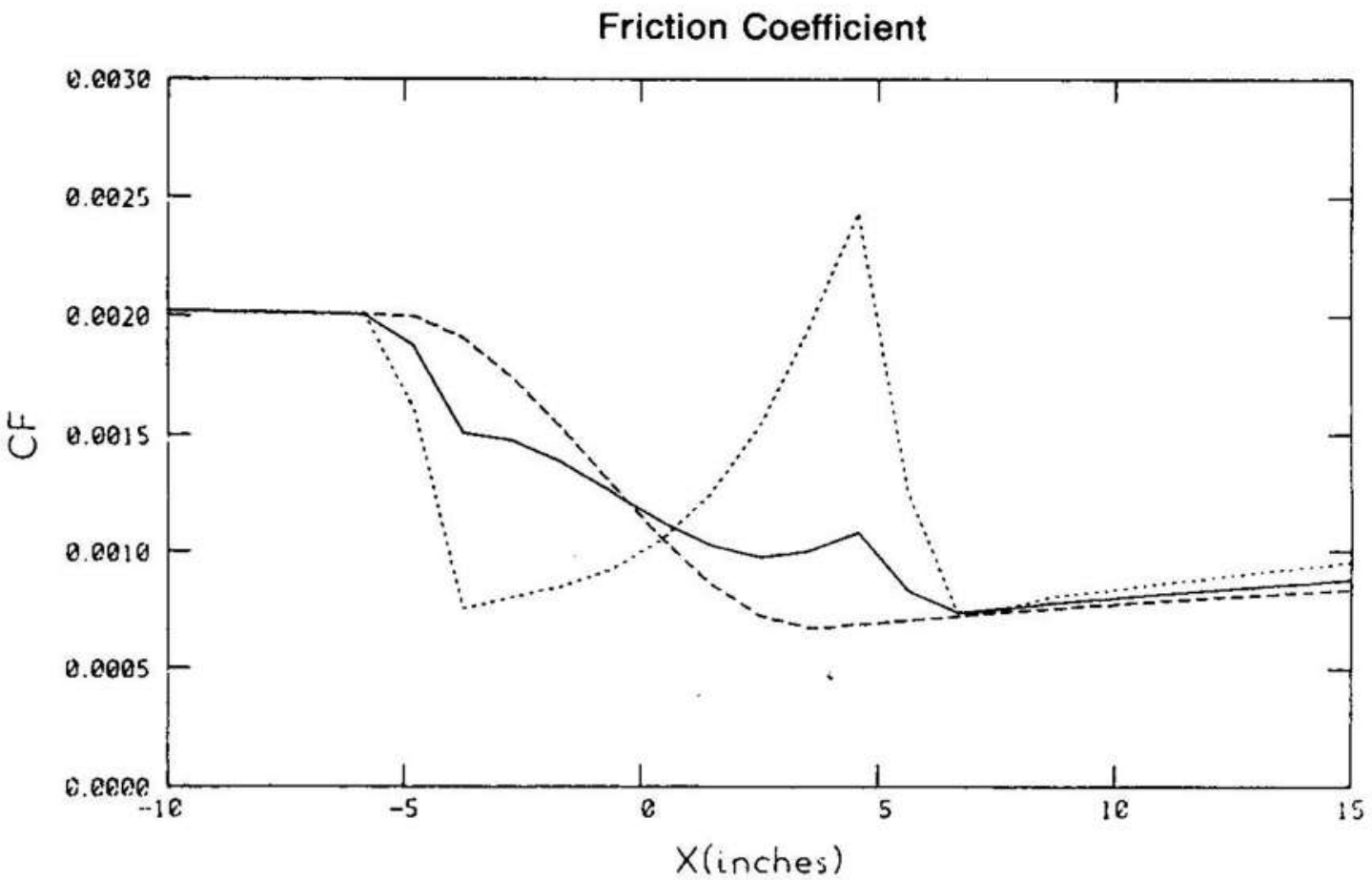

Figure $6 \mathrm{~b}$

Results for a short stretch of porous surface, $M=1.37 .---, \sigma=0.0 ;-, \sigma=0.005 ; \cdots, \sigma=0.02$.

\section{Final remarks}

The complex problem of shock wave/boundary layer interaction has been studied using perturbation techniques. The approach used is such that, assuming that the flow has the structure shown in Fig. 1 and knowing the undisturbed profiles ahead of the shock, the solution for the several distinct regions follows 
naturally. Thus this analysis has the advantage of providing theoretical results without the need to resort to any experimental data.

As mentioned before, the complexity of the equations for the region downstream of the shock cannot be avoided since all the effects on the main flow due to the shock and the plenum chamber must be accounted for. Thus, the solution for the wall layer downstream the shock wave is particularly complicated since it is affected by the upstream injection, the normal shock wave and the downstream injection.

The author is indebted to Dr. L. C. Squire for his constant support and advice throughout the course of this research. $\mathrm{He}$ is also grateful to $\mathrm{Mr}$. N. Chokani for many constructive discussions. The summary in German was prepared by Dr. M. A. Heckl. This work has been financially supported by the Conselho Nacional de Desenvolvimento Cientifico e Tecnologico - CNPq (Brazilian National Research Council). Further financial support has been kindly provided by Darwin College and an ORS award.

\section{References}

[1] P. Krogmann, E. Stanewsky and P. Thiede, Effects of suction on shock/boundary layer interaction and shock induced separation. J. Aircraft 22, 37-42 (1985).

[2] H. T. Nagamatsu, R. D. Orozco and D. C. Ling, Porosity effects on supercritical airfoil drag reduction by shock wave/boundary layer control. AIAA paper No. 84-1682 (1984).

[3] H. T. Nagamatsu, R. V. Ficarra and R. Dyer, Supercritical airfoil drag reduction by passive shock wave/boundary layer control in the Mach number range 0.75 to 0.90 . AIAA paper No. 85-0207 (1985).

[4] C. R. Olling and G. S. Kulikravich, Porous aerofoil analysis using viscous-inviscid coupling at transonic speeds. Int. J. Num. Meth. in Fluids 7, 103-121 (1987).

[5] G. Savu, O. Trifu and L. Z. Dumitrescu, Supression of shocks on transonic aerofoils. 14th Int. Symp. on Shock Tubes and Waves, 92-101 (1983).

[6] A. F. Messiter, Interaction between a normal shock wave and a turbulent boundary layer at high transonic speeds. Part I: Pressure distribution, ZAMP 31, 204-227 (1980).

[7] M. S. Liou and T. C. Adamson, Jr., Interaction between a normal shock wave and a turbulent boundary layer at high transonic speeds. Part II: Wall Shear stress, ZAMP 31, 227-246 (1980).

[8] A. P. Silva-Freire, An asymptotic solution for transpired incompressible turbulent boundary layers. To be published in the Int. J. Heat \& Mass Transfer.

[9] A. P. Silva-Freire, An extension of the transpired skin-friction equation to compressible turbulent boundary layers. To be published.

[10] R. L. Simpson, Characteristics of turbulent boundary layers at low Reynolds numbers with and without transpiration. JFM 42, 769-802 (1970).

[11] T. N. Stevenson, A modified defect law for turbulent boundary layers with suction or injection. Cranfield College of Aero, Aero report No. 170 (1963).

[12] T. C. Adamson, Jr. and A. F. Messiter, Normal shock wave-turbulent boundary layer interactions in transonic flow near separation, Transonic flow problems in turbomachinery. Ed. T. C. Adamson Jr. and M. F. Platzer, 392-441 (1977).

[13] T. C. Adamson, Jr. and A. Feo, Interaction between a shock wave and a turbulent boundary layer at transonic speeds, SIAM J. Appl. Math. 29, 121-145 (1975).

[14] G. E. Gadd, Interactions between shock waves and turbulent boundary layers, ARC R \& M 3262 (1961).

[15] W. G. Sawyer and C. J. Long, A study of normal shock-wave turbulent boundary layer interaction at Mach numbers 1.3, 1.4 and 1.5, RAE TR No. 82099 (1982). 


\section{Summary}

In this work perturbation techniques are used to study the problem of the interaction between a shock wave and a transpired turbulent boundary layer at transonic speeds. In the case considered here, the Mach number is assumed to be high enough for the sonic line to penetrate deep into the boundary layer so that it ends close to the wall. The flow region is divided into a region of strong interaction and regions of weak interaction. For the regions of weak interaction, upstream and downstream of the shock, a classical two-deck structure is assumed to hold for the boundary layer. Solutions chosen for these regions must account for the effects of blowing or suction. The strong interaction region on the other hand is shown to consist of three decks. A detailed analysis of the whole flow field is carried out and solutions valid in the double limit as Reynolds number tends to infinity and Mach number tends to one are proposed. Solutions of adjacent layers are shown to match so providing a smooth solution for the entire flow region. The analysis yields solutions for the pressure and skin-friction profiles.

\section{Zusammenfassung}

In dieser Veröffentlichung wird mit Methoden der Störungsrechnung das Problem der Wechselwirkung zwischen einer Schockwelle und einer turbulenten Grenzschicht mit Blas- und Saugeffekten beim Übergang zu Überschallgeschwindigkeiten untersucht. In dem hier betrachteten Fall ist die Machzahl so hoch, daß die Schockwellenfront tief in die Grenzschicht eindringt und nahe bei der Wand endet. Das Strömungsgebiet wird in ein Gebiet starker Wechselwirkung und in ein Gebiet schwacher Wechselwirkung eingeteilt. Für die Gebiete schwacher Wechselwirkung (vor und hinter der Schockwellenfront) wird angenommen, daß die Grenzschicht eine klassische zwei-Deck Struktur besitzt. Lösungen, die für diese Gebiete gewählt werden, müssen die Blas- oder Saugeffekte erklären. Es wird gezeigt, daß das Gebiet starker Wechselwirkung dagegen aus drei Decks besteht. Eine ausführliche Berechnung des gesamten Strömungsfeldes wird durchgeführt, und es werden Lösungen angegeben, die gültig sind für den doppelten Grenzfall, daß die Reynoldszahl gegen unendlich und die Machzahl gegen eins strebt. Es zeigt sich, daß die Lösungen für benachbarte Schichten aneinander passen, und daß sich damit eine stetige Lösung für das gesamte Strömungsgebiet ergibt. Die Lösungen für die Druck- und Wandreibungsprofile werden bestimmt.

(Received: October 23, 1987) 